OPEN ACCESS

Edited by: Masaki Shiota,

Kyushu University, Japan

Reviewed by:

Kenjiro Imada,

Kyushu University, Japan

Hiroaki Matsumoto,

Yamaguchi University, Japan

*Correspondence:

Xuesen Dong

xdong@prostatecentre.com

Specialty section:

This article was submitted to

Genitourinary Oncology,

a section of the journal

Frontiers in Oncology

Received: 02 October 2018 Accepted: 26 November 2018 Published: 07 December 2018

Citation:

Chen R, Li H, Li Y, Fazli L, Gleave M, Nappi $L$ and Dong $X$ (2018) Loss of Nuclear Functions of HOXA10 is

Associated With Testicular Cancer Proliferation. Front. Oncol. 8:594. doi: 10.3389/fonc.2018.00594

\section{Loss of Nuclear Functions of HOXA10 Is Associated With Testicular Cancer Proliferation}

\author{
Ruiqi Chen ${ }^{1,2}$, Haolong $\mathrm{Li}^{1}$, Yinan $\mathrm{Li}^{1}$, Ladan Fazli ${ }^{1}$, Martin Gleave ${ }^{1}$, Lucia Nappi ${ }^{1}$ and \\ Xuesen Dong ${ }^{1 *}$ \\ 1 Department of Urologic Sciences, Vancouver Prostate Centre, The University of British Columbia, Vancouver, BC, Canada, \\ ${ }^{2}$ Department of Medicine, King's College Circle Toronto, University of Toronto, Toronto, ON, Canada
}

Background: HOXA10 is a key transcriptional factor that regulates testis development as reported from previous transgenic mouse models and human inherited diseases. However, whether it also plays important roles in promoting the development of testicular cancer is not well-understood.

Objective: To study the expression of HOXA10 and its regulated signaling pathways in testicular cancers.

Design, Setting, and Participants: A tissue microarray was constructed with benign and cancerous testis. TCam2, NT-2, and NCCIT cell models were applied in this study.

Intervention: Immunohistochemistry and immunofluorescence were performed to measure the expression and cellular localization of HOXA10 in testicular cancer tissues and cell models. Cell proliferation and cell cycling rates were determined by BrdU incorporation and flow cytometry assays. HOXA10 transcriptomes were profiled with Ampliseq RNA-seq in testicular cancer cells. Immunoblotting assays were used to detect HOXA10-regulated signaling.

Results: HOXA10 is a nuclear protein in benign spermatocytes. Reduced nuclear expression and increased cytoplasmic expression of HOXA10 are associated with testicular cancers. These changes are consistent in both seminoma and non-seminoma. Enhanced HOXA10 expression in testicular cancer cell models inhibits cell proliferation and delays cell cycle progression through G2/M phases. These functions of HOXA10 mainly affect the TP53, cKit, STAT3, AKT, and ERK signaling pathways.

Conclusions: Loss of nuclear functions of HOXA10 enhances proliferation of testicular cancer cells, suggesting that downregulation of HOXA10 transcription activity may promote the development of testicular cancers.

Keywords: testicular cancer, testicular germ cell tumor, HOXA10, TP53, proliferation

\section{INTRODUCTION}

Testicular germ cell tumor (TGCT) is the most common form of cancer in young men aged between 15 and 40 and the incidence has been increasing over the past few decades worldwide (1-3). Despite a relatively high cure rate of $95 \%$ in all TGCT patients upon diagnosis and $80 \%$ in patients with metastatic TGCT, over 10,000 deaths worldwide were estimated in men with this disease (WHO GLOBOCAN 2012). Unlike spermatocytes, TGCT likely develops from gonocytes that failed to 
differentiate into spermatogonia, thus showing similar patterns of gene expression to those of stem cells and intratubular germ-cell neoplasm (4). TGCT can be categorized into seminoma, nonseminoma and spermatocytic tumors (5). Seminomas resemble closely with intratubular germ-cell neoplasia in a blocked differentiation state. Non-seminomas include several histological subtypes such as embryonal carcinoma, choriocarcinomas, yolksac tumors, and teratoma. Of note, choriocarcinomas and yolksac tumors present with extraembryonal differentiations while teratomas show somatic differentiations that contain all three germ layers.

Although previous studies have identified some genetic and epigenetic aberrations associated with TGCT, one group of genes that may play important roles in this process is the homeobox gene family. The homeobox gene family consists of 37 genes that encode transcription factors to regulate morphogenesis and differentiation of cells during embryogenesis, and are frequently deregulated in various types of cancer (6). These genes are crucial in the development of testis and expressed in human testis throughout adulthood (7). Importantly, TGCT tumorigenesis resembles early embryogenesis in many aspects ranging from gene expression patterns $(8,9)$, imprinting patterns $(10,11)$, and response to in vitro differentiation stimulus (12). Moreover, a previous epigenetic study showed that the promoter of some homeobox genes such as RASSF1A, $S C G B 3 A 1$, and HOXA9 were hypermethylated in testicular cancer tumors (13), further supporting that deregulation of homeobox proteins may contribute to the development of TGCT. Among the homeobox family genes, aberrant HOXA10 expressions have been implicated in numerous other types of cancers but not yet described in TGCT. HOXA10 is a member of the abdominal $B$ class that also consists of HOXA9 and HOXA13 (14). Like other HOX family proteins, the HOXA10 protein is known to be localized in the nucleus and binds to DNA via a consensus core of TTAT/TTAC that is influenced by flanking sequences (15), interacting proteins such as MEIS and $\operatorname{PBX}(16,17)$, and coregulatory proteins like histone deacetylase 2 (18). In the setting of cancers, HOXA10 deregulation is known to play significant roles in mammary carcinoma, endometrial carcinoma, head and neck squamous cell carcinoma (HNSCC). Interestingly, the roles of HOXA10 are complex among different types of cancers. For example, HOXA10 overexpression promotes endometrial cancer and HNSCC activities (19, 20), whereas HOXA10 inhibition is associated with breast cancer tumorigenesis (21). However, the role of HOXA10 in TGCT has not yet been elucidated. In this study, we have combined tumor histological explorations, transcriptomic studies in cell lines, and functional investigations to characterize HOXA10 expression and function in TGCT tumorigenesis.

\section{MATERIALS AND METHODS}

\section{Human Testicular Samples}

Human testicular tissue samples were obtained from the Vancouver Prostate Center (VPC) tissue bank at the University of British Columbia. Patient information is listed in Table S1.
All patients have signed an informed consent to a protocol that was reviewed and approved by the UBC Clinical Research Ethics Board (Certificate \#: H09-01628).

\section{Immunohistochemistry}

Whole sections of testicular samples were fixed in $10 \%$ neutral buffered formalin, embedded in paraffin, stained with $\mathrm{H} \& \mathrm{E}$, and evaluated by a pathologist (L.F.) for benign and cancerous portions of the testes. A tissue microarray (TMA) was also constructed, as previously described (2224). Immunohistochemistry assays were performed by Ventana Discovery XT autostainer (Ventana). Slides in citrate buffer ( $\mathrm{pH}$ $=6$ ) were heated in a steamer for $30 \mathrm{~min}$. After cooling for $30 \mathrm{~min}$ and washing, the slides were incubated in 3\% $\mathrm{H} 2 \mathrm{O} 2$ for $10 \mathrm{~min}$, blocked with $3 \%$ BSA for $30 \mathrm{~min}$, and then incubated with indicated primary antibodies for $2 \mathrm{~h}$ at room temperature. The slides were washed extensively with PBS and examined with UltraMap kit (Ventana). The sections were counterstained with hematoxylin and mounted with coverslips using the xylenebased mounting medium, Cytoseal (Stephen Scientific, Riverdale, NJ). Normal IgG antibodies (Santa Cruz) were used as negative controls. Information on HOXA10 and AR antibodies used in this study is listed in the Supplementary Materials. Stained slides were scanned by a Leica SCN400. Digital images were evaluated and scored by the pathologist (L.F), based on subcellular localization, intensity and percentile of positive cells within a tissue core. Digital images were evaluated by Dr. Ladan Fazli as well as by using the software, Image Pro Plus (Media Cybernetics Inc), to score the percentage of stained cells $(0-16,17-33,34-$ 66 , and $67-100 \%$, as $0-3$ scores) and the staining intensity (no staining, low, moderate, and high intensity staining, as $0-3$ scores). The histology index of HSCORE $=\sum \mathrm{pi}(i+1)$, where $i=$ the intensity of staining and $\mathrm{pi}=$ the percentage of stained cells as reported (22-24).

\section{Immunofluorescence Microscopy}

Immunofluorescence assays were performed as previously described (25). Slides were deparaffinized, rehydrated through a series of graded alcohols, and washed in double deionized water for $5 \mathrm{~min}$. Tissues were then placed in antigen unmasking solution (Vector Labs, Burlingame, CA), and antigen retrieval was performed by microwaving samples. Slides were cooled to room temperature, and washed with $\mathrm{PBS}$ ( $\mathrm{pH}$ 7.4). For sequential immunofluorescence staining, the AR (N-20) antibody (\#sc-816, Santa Cruz) at 1:50 dilution was first used, followed by HOXA10 (ab191470, Abcam) at 1:100 dilution on the slides. It is followed by 1:500 Alexa Fluor 568 Donkey anti-rabbit IgG (cat\#A10042, Invitrogen) and 1:500 Alexa Fluor 488 Donkey anti-Goat IgG (cat\#A11055, Invitrogen) secondary antibodies applied to the slides. Slides were counterstained and mounted with coverslips using Vectashield mounting medium containing $4^{\prime}, 6^{\prime}$-diaminido-2-phenylindole (DAPI; Vector Laboratories, Burlingame, CA, USA). Images were captured using a Zeiss LMS780 Confocal microscope (Carl Zeiss Instruments, Oberkochen, Germany) at 40X magnifications. 


\section{Cell Culture and Lentivirus Infection}

Three testicular cancer cell lines, TCam2, NT-2 and NCCIT, were generously provided by Dr. Leendert H. J. Looijenga (University Medical Center Rotterdam, Netherlands). Cell culturing methods followed the protocol described by Dr. Looijenga group (26). Lentiviurs vectors encoding control or HOXA10 were constructed using the FUGWBW vector as a backbone (25, 27-29). Invitrogen gateway system was used to package lentivirus. Briefly, $3 \mu \mathrm{g}$ of each lentiviral vector ( $p$ FUGWBW mock vector, Flag-HOXA10) together with $9 \mu \mathrm{g}$ of the ViraPower packaging mix (Invitrogen) were transfected into $293 \mathrm{~T}$ cells, using Lipofectamine 3,000 at $37^{\circ} \mathrm{C}$ and $5 \%$ $\mathrm{CO} 2$. Lentiviral particles were harvested by removing medium $48 \mathrm{~h}$ after transfection and used to infect TCam2, NT-2, and NCCIT cell lines. Lentivirus transduced cells were then exposed to $10 \mathrm{ng} / \mathrm{ul}$ blasticidin to select stably infected cells for 3 weeks. Polyclonal and antibiotic resistant cells were pooled for further experiments.

\section{Whole Transcriptome Profiling}

Total RNA was extracted from TCam2 cells infected with HOXA10 or control by using the mirVana RNA Isolation Kit (Ambion, Burlington, Canada) according to the manufacturer's protocol. Subsequent sequencing and analysis were described previously (30). In summary, the quantity and quality of the RNA samples were assessed by Nano-drop 2,000 as well as Agilent 2,100 Bio-analyzer (Caliper Technologies Corp., Canada) before sent for AmpliSeq Transcriptome Sequencing. Library preparation, sequencing, and primary analyses were performed by the UBC-DMCBH Next Generation Sequencing Center following the protocol described by $\mathrm{Li}$ et al. (31). Primary and differential gene expression analyses were carried out by the AmpliSeq RNA plugin available through the Ion Torrent $^{\mathrm{TM}}$ suite Software (31) and the R/Bioconductor package DESeq2 (32).

\section{BrdU Incorporation and Cell Cycling Assays}

Cell proliferation rates were measured by using the bromodeoxyuridine (BrdU) proliferation assay kit (Millipore, Catalog \# 2750) according to the manufacturer's protocol and as previously described (29). Cell cycling was analyzed by FACS with $40 \mathrm{ug} / \mathrm{mL}$ propidium iodide staining following the protocol http://www.meduniwien.ac.at/user/johannes.schmid/ PIstaining3.htm. Relative DNA contents from 10,000 cells were analyzed by FACSCanto II flow cytometer and BD FACSDiva software v5.0.3 (Becton Dickinson) as we reported (25).

\section{Western Blotting}

Cells were lysed in buffer [50 mM Tris- $\mathrm{HCl}, \mathrm{pH} 7.4 ; 10 \mathrm{mM}$ EDTA; 5 mM EGTA; 0.5\% NP40; 1\% Triton X-100 plus protease inhibitor (Roche)]. Protein concentration was measured by Pierce BCA protein assay kit (Thermo). Protein samples (40-60 ug) were mixed with SDS-PAGE loading dye, boiled and loaded on SDS-PAGE gel. After transferring onto PVDF membranes, proteins were blotted with primary antibody in TBST $(50 \mathrm{mM}$
Tris/pH 7.5, 0.15 M NaCl, and 0.05\% Tween-20) plus 5\% fatfree milk, washed and then with the HRP-conjugated IgG for $1 \mathrm{~h}$. Membranes were then treated with ECL reagent (GE healthcare) and exposed to $\mathrm{x}$-ray film. Vinculin, tubulin or histone $\mathrm{H} 3$ was used as a loading control. Antibody and primer information were included in Table S2.

\section{RESULTS}

\section{HOXA10 Expression in Testicular Tissues}

To investigate the cellular localization of HOXA10 protein in testis tissue, the whole sections of benign testis slides were incubated with a HOXA10 antibody and HOXA10 expression was evaluated by immunohistochemistry (IHC). Within the seminiferous tubule, HOXA10 was highly expressed in the nuclei of spermatocytes at various differentiation stages (Figure 1A). In contrast, Sertoli cells were HOXA10 negative. Since Sertoli cells are often difficult to be distinguished from spermatocytes, we used the androgen receptor (AR) as a Sertoli cell-specific marker. Co-immunofluorescence assays with both HOXA10 and AR antibodies (Figure 1B) showed that HOXA10 (green) was not co-localized with the AR (red) in cells within the seminiferous tubules. Interestingly, HOXA10 was expressed in the cytoplasm of Leydig cells and absent in the smooth muscle cells surrounding the seminiferous tubules. On the same slides, HOXA10 was expressed at a relatively high level in the cytoplasm of the columnar cells and basal cells within the pseudostratified columnar epithelium of the epididymis, whereas the cells within the connective tissue underneath pseudostratified columnar were HOXA10 negative (Figure S1). These IHC results indicate that HOXA10 protein is expressed specifically in the nuclei of the spermatocytes (germline cells), while absent or localized in the cytoplasm of somatic cells including the Leydig cells in the testis and the pseudostratified columnar epithelial cells in the epididymis. Since HOXA10 is widely known as a transcriptional factor and our results show that HOXA10 is only localized in the nuclei of spermatocytes, this protein may be crucial in regulating the normal course of spermatocyte development and differentiation.

\section{Reduced Nuclear Expression of HOXA10 Protein in Testicular Cancers}

To study the expression of HOXA10 in testicular tumors, we applied IHC on a tissue microarray (TMA), containing 5 benign, 96 seminoma, 8 spermatocytic tumor and 17 non-seminoma (Figure 2). While highly expressed in spermatocytes of benign testes $($ HScore $=1.98+0.15$ ), all cancerous testes had extremely low or no nuclear HOXA10 expression with HScores $=0.067$ +0.019 in seminoma, $0.207+0.06$ in spermatocytic tumor, and 0 in non-seminoma. In contrast, cytoplasmic HOXA10 was mainly expressed in testicular cancer cells (HScore $=1.40$ +0.06 in seminoma, $1.67+0.167$ in spermatocytic tumor and $1.45+0.13$ in non-seminoma), but less commonly in benign testis (HScore $=0.28+0.07$ ). We have also performed RNA in situ hybridization (RISH) assays using a HOXA10specific RNA probe on the same TMA. We observed that both benign and cancerous testis tissues expressed HOXA10 RNAs 


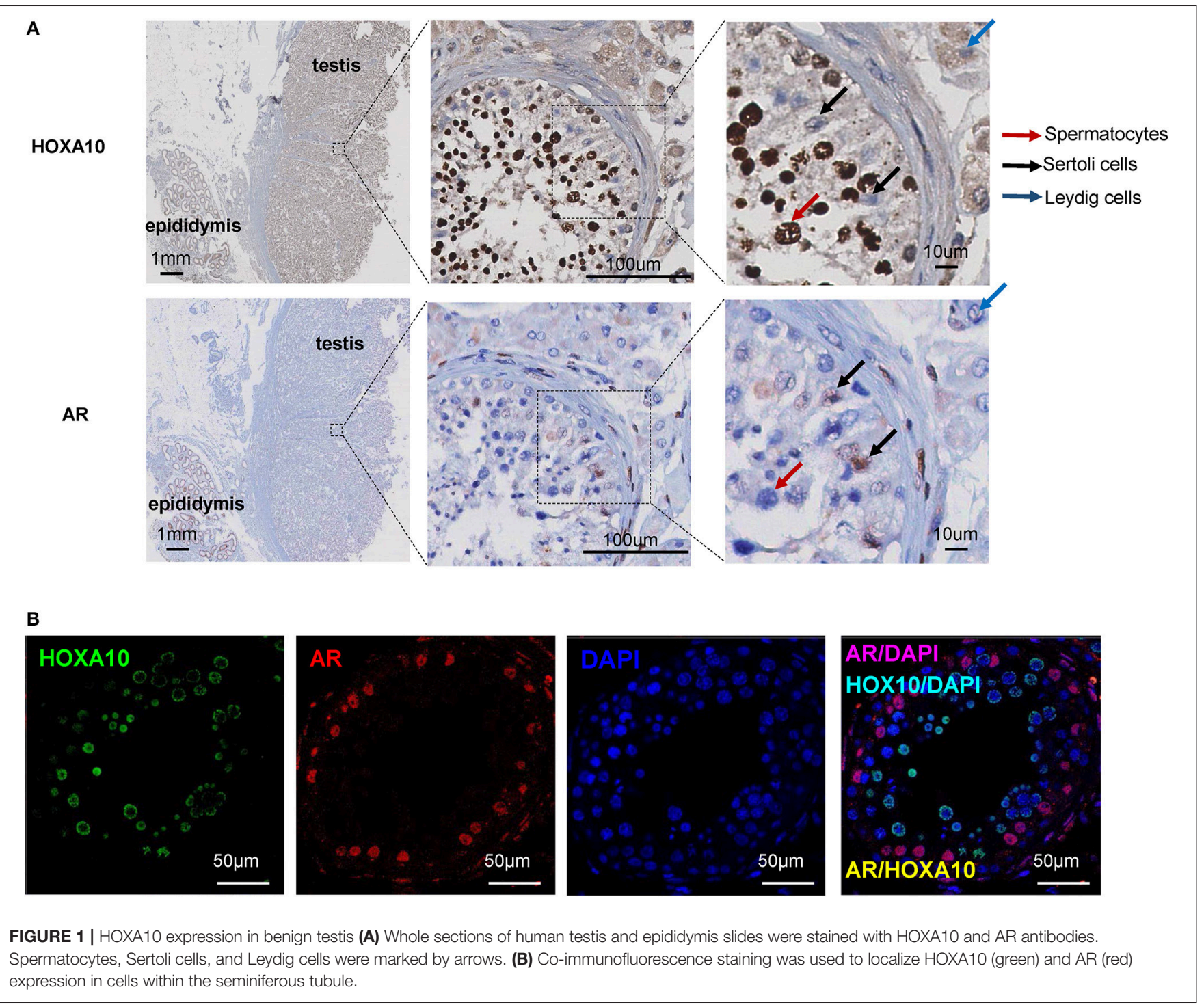

and there were no differences between benign and cancer tissue samples (Figure S2). Together, these results indicate that loss of nuclear expression of HOXA10 is associated with testicular cancers.

\section{Profiling HOXA10 Transcriptome in Testicular Cancer Cells}

To study the cellular functions of HOXA10 in testicular cancer cells, we used three available TGCT cell lines, TCam2, NT-2, and NCCIT. While all cell models were HOXA10 negative at both mRNA and protein levels (Figure S3), exogenous HOXA10 localized only in the nuclei. These results were consistent with our IHC observations that showed loss of nuclear expression of HOXA10 in all subtypes of testicular tumors. However, the differences of cytoplasm expression of HOXA10 between tumor cells and cell lines may reflect their different in vivo and in vitro growth conditions. Nevertheless, the expression pattern between tumor cells and in vitro cell models support that loss of nuclear function of HOXA10 is associated with testicular cancer.

Since seminoma accounts for approximately half of testicular cancers, we used the TCam2 testicular seminoma cell line as a model to further characterize HOXA10 transcriptome in testicular cancer cells. Using the AmpliSeq Transcriptome Sequencing method, we have identified 1,543 significantly upregulated genes and 839 downregulated genes by HOXA10 (fold-change cutoff $>1.5$ and padj $<0.05$ ). The 30 top ranked HOXA10-regulated genes by fold change are listed in Figure 3A. DAVID (Database for Annotation, Visualization, and Integrated Discovery) analyses indicated that overexpression of HOXA10 was associated with gene ontology (GO) terms such as cell signal transduction, regulation of cell proliferation, cell cycling, cellular movement, and adhesion (Figure 3B). These results suggest that aberrant HOXA10 expression may interrupt typical spermatocyte differentiations through altering cell cycles and cell proliferation, resulting in the development of TGCT. Similarly, 


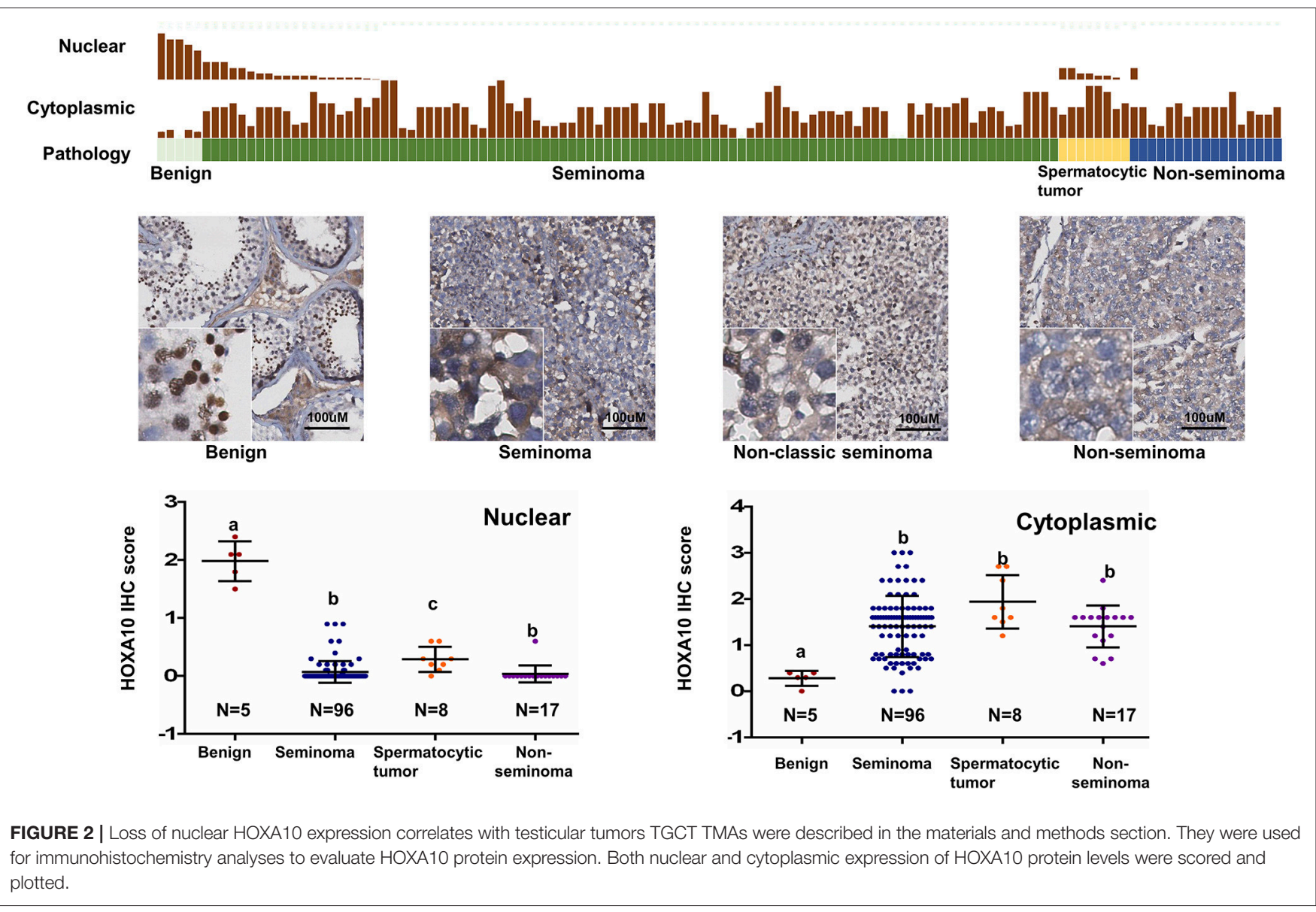

Ingenuity Pathway Analysis (IPA) predicted that the upstream regulators of HOXA10 included increased IFN $\alpha$, STAT1, TP53 signaling and decreased STAT3 signaling, all of which have been implicated in tumor development (Figure 3C). We further applied Gene Set Enrichment Analysis (GSEA) to show that HOXA10 transcriptome in TGCT cells was positively associated with TP53 pathway $(\mathrm{NES}=1.95, \mathrm{FDR} q<0.001)$ while negatively associated with $\mathrm{G} 2 / \mathrm{M}$ checkpoint $(\mathrm{NES}=-1.81, \mathrm{FDR} q<0.007)$ (Figure 3D). These results support that HOXA10 suppresses TGCT cell proliferation through the modulation of the TP53 signaling. Based on these results, we hypothesize that HOXA10 can enhance the functions of TP53 to suppress TGCT cell proliferation.

\section{HOXA10 Suppresses Testicular Cancer Cell Proliferation}

To confirm the transcriptomic analyses results, we performed BrdU incorporation assays to show that HOXA10 overexpression suppressed cell proliferation of all three TGCT cell models. These results suggested that our findings from the transcriptomic studies in TCam 2 cell may also be applied to the non-seminoma NT-2 and NCCIT cell models (Figure 4A). To delineate the specific cell cycling stages regulated by HOXA10, we performed FACS assays on TCam2, NT-2, and NCCIT cells (Figure 4B).
Cells expressing control or HOXA10 were first serum starved to synchronize cell cycling at the G0/G1 stage, followed by adding culture medium containing $10 \%$ serum to release cell cycling. We observed that HOXA10 has no impact on cells passing from the G1 to S and G2/M phases. However, when cell cycling was synchronized at the G2/M phases by nocodazole and then released, HOXA10 caused significant delays of cell cycling passing through the G2/M phases into the G1 phase. These results indicate that HOXA10 inhibited TGCT cell proliferation through impeding the G2/M progression.

\section{HOXA10 Mediates Multiple Signaling to Regulate Testicular Cell Proliferation}

To further confirm the predicted HOXA10 functions by transcriptomic analyses and to better understand the signaling pathways regulated by HOXA10, we used immunoblotting assays in the three TGCT cell models. First, we observed that HOXA10 transfection induced TP53 expression (Figure 5A). Since TP53 mediates its tumor-suppressive activities through regulating gene transcriptions, we found that HOXA10-induced TP53 was localized in the nuclei via immunoblotting nuclear extracts of cells and immunofluorescence microscopy assays (Figure 5B). Furthermore, upregulation of TP53 by HOXA10 was concurrent with the induction of cyclin-dependent kinase inhibitor p21, 


\section{A TCam2 (HoxA10) vs TCam2 (Mock)}

Top 30 genes with fold change (log2 value)
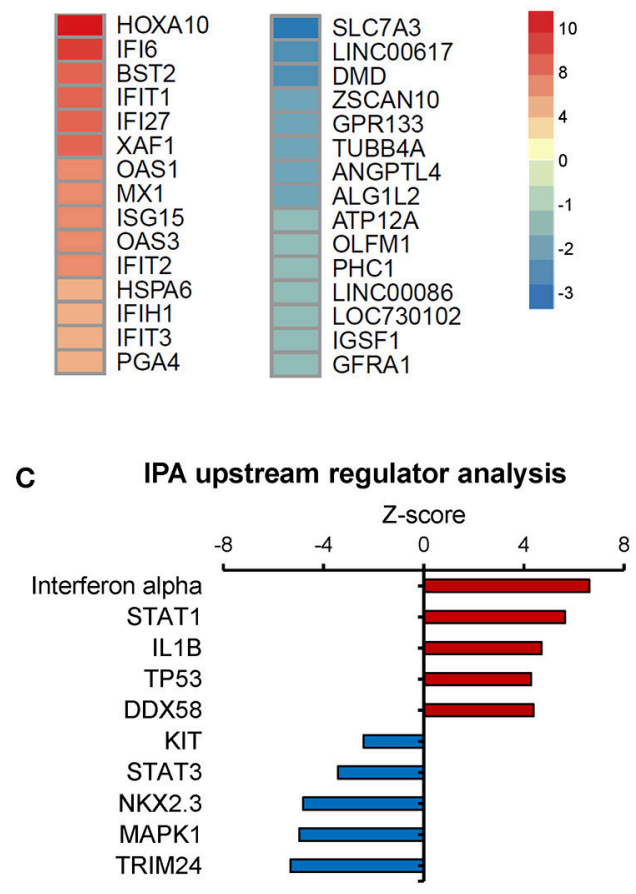

B

TCam2 (HoxA10) vs TCam2 (Mock)
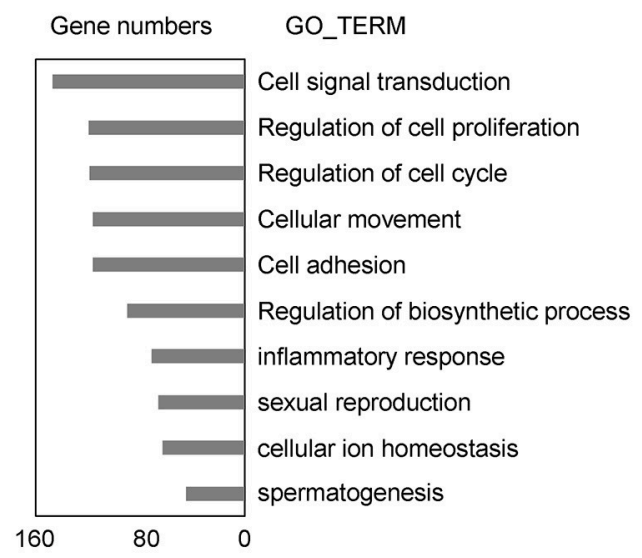

D

Gene set enrichment analysis (GSEA)
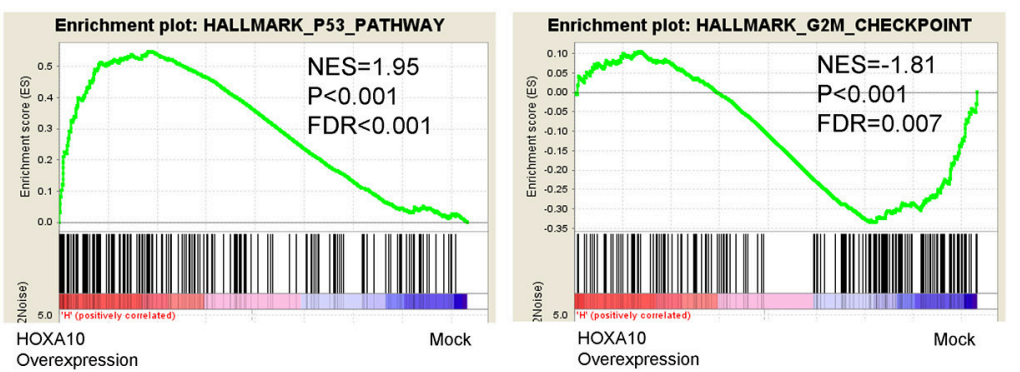

FIGURE 3 | HOXA10 transcriptome in testicular cancer cells (A) A heatmap presents the top-ranked 30 genes that are transcriptionally regulated by HOXA10 in TCam2 cells. (B) Differentially expressed genes by HOXA10 were subjected to DAVID (version 6.7) analyses. Top ranked GO_TERM groups by $p$-value from the smallest to the largest are listed. (C) IPA predicts the upstream regulators of HOXA10 transcriptome. Red and blue nodes indicate signaling that is either stimulated or inhibited according to the Z-score. (D) HOXA10 transcriptomes were analyzed by the DESeq2 package in R. GSEA enrichment plots showed positive correlations of HOXA10-regulated genes with the TP53 pathway (normalized enrichment score (NES) $=1.95$, false discovery rates (FDR) $<0.001$ ) and negative correlation with the G2/M checkpoint (normalized enrichment score $(\mathrm{NES})=-1.81$, false discovery rates $(\mathrm{FDR})=0.007$ ).

but not p27 (Figure 5A). These findings are consistent with the relationship between p21 and TP53, whereby TP53-mediated growth inhibition is dependent on the induction of p21 to suppress cell cycle progression (33). Interestingly, cyclin D1 protein levels were reduced by HOXA10 in NT-2 and NCCIT but not the TCam2 cells, while cyclin E expression was suppressed by HOXA10 in TCam 2 and NCCIT, but not the NT-2 cells. Nevertheless, HOXA10 stimulates the TP53 and putatively the TP53-p21 axis, thereby exerting general inhibitory effects to all TGCT cell models regardless the inconsistent changes in cyclin $\mathrm{D}$ and cyclin E expressions.

In addition to confirming the TP53 signaling, we also performed immunoblotting assays to validate the relationship between HOXA10 and cKit as well as STAT3 signaling pathways (Figure 5C). The protein cKit is a receptor tyrosine kinase generally localized on the cell surface. It can be activated by the stem cell factor, SCF, to trigger the downstream signaling to stimulate cell proliferation, survival, and migration (34). cKit was expressed in both TCam 2 and NCCIT cells and its expression was suppressed by HOXA10. Moreover, the functionally active form of STAT3 (phosphorylated STAT3, pSTAT3) was observed in the three testicular cell lines, with relatively higher levels in the NCCIT cells. HOXA10 reduced pSTAT3 levels in the NCCIT cells, and abolished pSTAT3 expression in both TCam2 and NT-2 cells. Furthermore, phosphorylated AKT and Erk kinases as the downstream effectors of the cKit and MAPK1 pathways were also inhibited by HOXA10. These results indicate that HOXA10 not only stimulates the TP53-p21 axis, but also suppresses the cKit and STAT3 pathways to inhibit testicular cell proliferation (Figure 5D), supporting that loss of HOXA10 functions is associated with testicular cancers.

\section{DISCUSSION}

In this study, we report that HOXA10 is expressed in the nucleus of benign spermatocytes (Figure 1) and frequently loses its nuclear localization in TGCTs including both seminoma and non-seminoma (Figure 2). Traditionally, HOXA10 is considered a nuclear protein that functions as a transcription factor to regulate downstream gene expressions. To date, only 


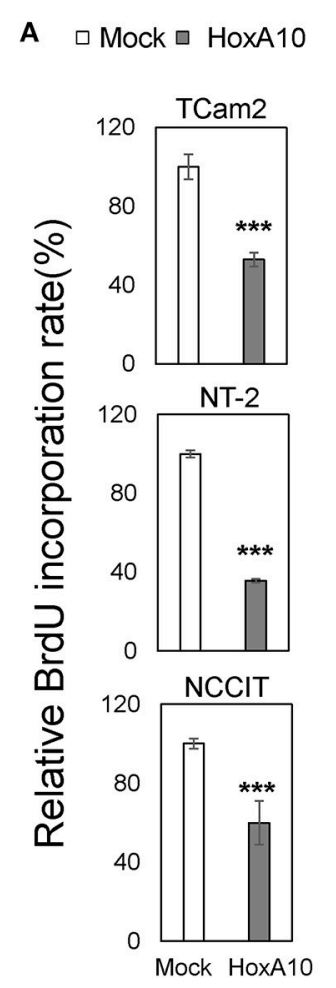

\section{B $\square$ Mock $\square$ HoxA10 Release from serum starve Release from Nocodazole}

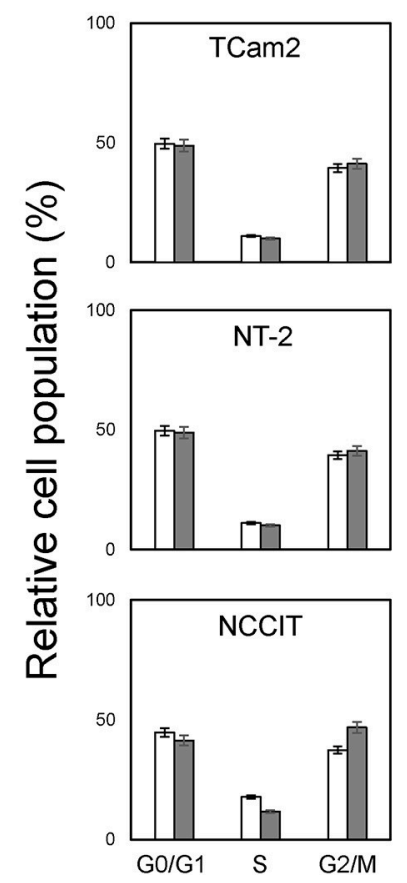

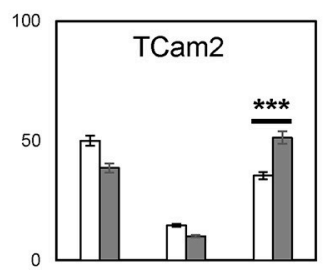
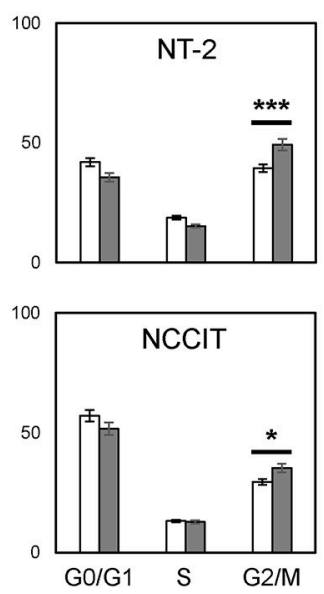

FIGURE 4 | HOXA10 inhibits testicular cell proliferation (A) TCam2, NT-2, and NCCIT cell lines with control or HOXA10 overexpression were used to perform BrdU incorporation assays to measure cell proliferation rates. BrdU results represent colorimetric quantitative measurements (OD at $450 \mathrm{~nm}$ ) of cellular BrdU incorporation into DNA. ${ }^{\star} p<0.05$ and ${ }^{\star \star *} p<0.001$. (B) TCam2, NT-2, and NCCIT cells with control or HOXA10 overexpression were synchronized by serum starvation for $12-16 \mathrm{~h}$ and then either released from serum starved conditions (left); TCam2, NT-2, and NCCIT cells were synchronized by Nocodazole, then released for cell cycling progression (right). Cells were collected and subjected to FACS assays to measure cell populations at the G0/G1, S, and G2/M phases.

one group reported cytoplasmic localization of HOXA10 via interacting with the histone deacetylase Sirtuin2 (SIRT2) (35). This report suggested that SIRT2 can bind with HOXA10 in the cytoplasm to suppress HOXA10-mediated transcription. Similarly, we also observed extensive cytoplasmic HOXA10 expressions from IHC staining in TGCT patient samples (Figure 2), despite similar levels of RNA expressions between benign and cancerous tissues as further cross-validated with the RISH procedure (Figure S2). Specific mechanisms leading to this change in HOXA10 localization remain elusive. One possible explanation may be due to increased SIRT2 that sequesters HOXA10 in the cytoplasm. In addition, since HOXA10 tyrosine phosphorylation was reported to have reduced its DNA-binding activities (36), disruptions in HOXA10 phosphorylation regulators such as JAK2 (37) and phosphatases SHP1/SHP2 may contribute to the cytoplasmic localization (38). Other putative explanations include reduced microRNAs that regulate HOXA10 expression and genomic mutations that affect HOXA10 nuclear localization sequences. These two theories are unlikely to be true as the former should lead to significant change in HOXA10 protein abundance inconsistent with our observations in this study and the latter has not been reported from recent genome-wide association studies (GWAS) (39).
Changes in HOXA10 localization in TGCT has functional significances. Our transcriptomic studies showed that overexpression of "functional" HOXA10 in the nucleus of TCam2 cells (Figure S3) led to changes in the differential expression of numerous genes (Figures 3A-C), notably the stimulation of TP53 pathway and the suppression of G2/M checkpoint signaling (Figures 3C,D). These associations were confirmed with our molecular analyses (Figures 4, 5A,B), suggesting an anti-proliferative property of HOXA10 in our cell models. For the TP53 signaling, previous reports have shown that not only TP53 promoter contained HOXA binding sites, but also that HOXA10 was a positive regulator of TP53 in breast cancer cells (21). Interestingly, this anti-tumorigenic HOXA10TP53 relationship has only been described in breast cancer but not other types of cancer, raising the question of whether this pathway is only specific to endocrine or germ-related tumors. With regard to the G2/M checkpoint, the suppressive effect of HOXA10 on G2/M checkpoints may or may not be related to TP53. Although HOXA10-induced TP53 may directly inhibit $\mathrm{G} 2 / \mathrm{M}$ via cdc2 (40), it is possible that the suppressive effects of HOXA10 on other signaling pathways such as p21, cKit, STAT3, and MAPK also play a role in this process. The latter mechanisms are more likely to be true at least in NCCIT cells, which have mutated TP53 in contrast to that of TCam 2 and NT-2 


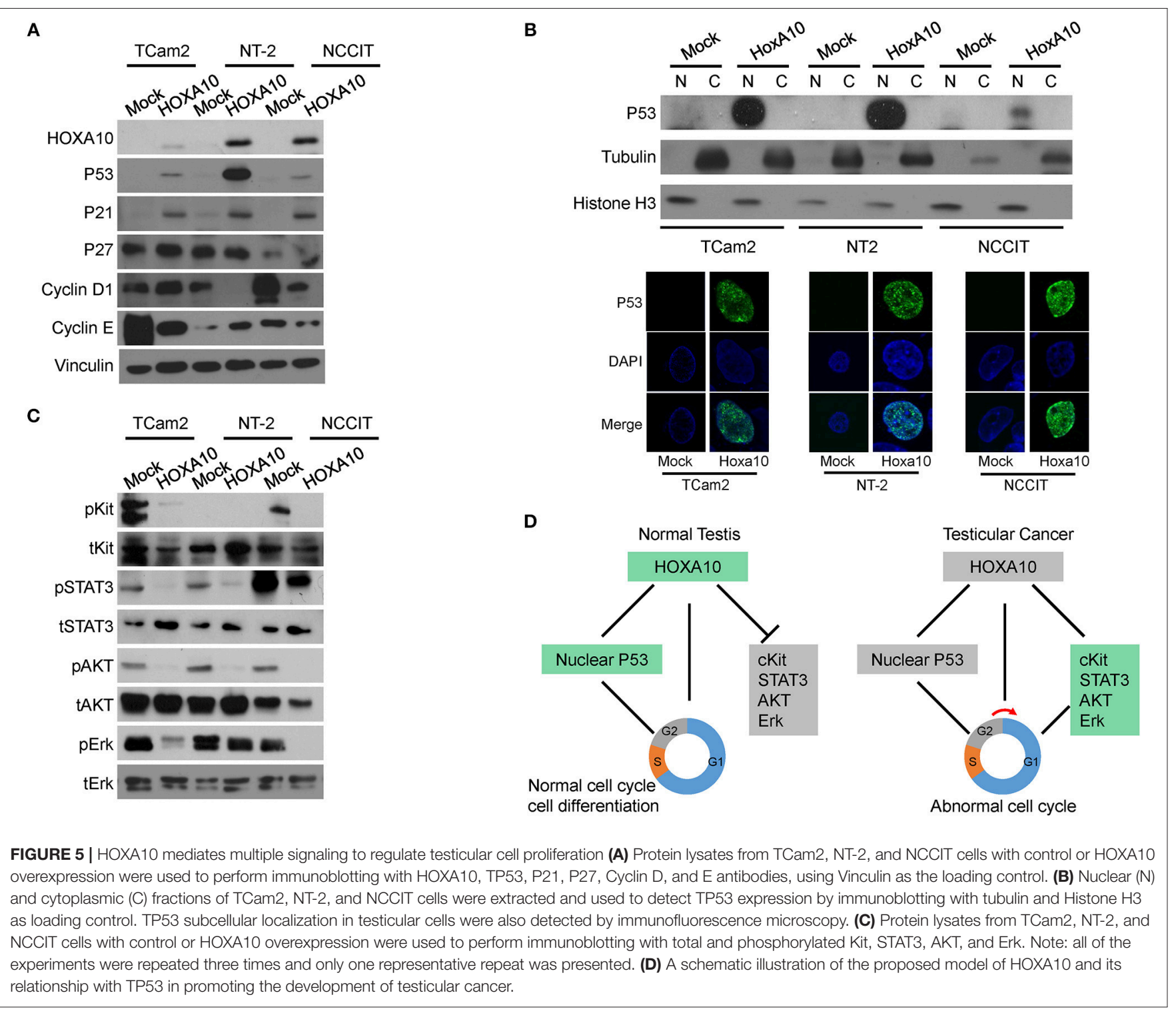

cells (41). In fact, as a transcription factor acting on numerous downstream effectors, whether HOXA10 directly or indirectly regulates signaling pathways involving proteins such as $\mathrm{p} 21$, cKit, STAT3, AKT, and Erk remains elusive. It is also possible that, in the setting of TGCT, the aberrant cytoplasmic localization could lead to new functional effects of HOXA10 in the cytoplasm. These hypotheses warrant further investigations. Loss of nuclear HOXA10 may also provide insights into differentiation in TGCTs. Somatic cancers usually develop via entering an undifferentiated state for clonal evolution and expansion. As a gene known to play important roles in development, HOXA10 is expected to affect gene expressions related to cell differentiation. However, TGCT is a special case as it is relatively undifferentiated and develops from the malignant counterpart of undifferentiated germ cells (e.g., gonocytes or primordial germ cells). A representative example is seminoma, which predominantly presents in a state of "blocked" differentiation
(8). As a result, (de)differentiation may play less critical roles than proliferation in the development of TGCT. In this context, our findings suggest that HOXA10 may have more impact on suppressing proliferation rather than regulating differentiation in anti-TGCT tumorigenesis. Nevertheless, since HOXA10 has numerous downstream effectors, it is also possible that HOXA10 is required for normal spermatocyte differentiation to prevent tumorigenesis. One limitation of this study might be related to the TGCT tumor models. We have used all three TGCT cell lines that are currently available. The TCam 2 cell line is the only one that resembles seminomas both in genomic and epigenetic changes (26). However, TCam2 cells have mutated BRAF genes uncommonly seen in TGCT tumors. While it was hypothesized that the constitutively active BRAF gene enables TCam 2 cells to be propagated in vitro, this aberration may interfere with cell growth and proliferation, particularly related to the RAS signaling pathway (e.g., Erk) (42). On the other hand, NCCIT 
cells resemble teratocarcinoma whereas NT-2 cells are derived from malignant embryonal carcinoma $(43,44)$. Comprehensive genetic and epigenetic characterizations of these cell lines may provide further insights into whether these models resemble the average non-seminomas.

Although our study is the first to report clinical and functional significances of HOXA10 in testicular cancer, it is interesting that HOXA10 mutations were reported to be linked to higher incidence of inguino-scrotal cryptorchidism $(45,46)$ and that cryptorchidism is one of the strongest risk factors for testicular cancer (7). In fact, although weaker, cryptorchidism remains a risk factor for TGCT even after early orchiopexy interventions (47). These finding, together, suggest that TGCT tumorigenesis likely happens both in utero and after birth, and that dysfunctional HOXA10 may aid the tumorigenic process throughout development. In addition, our findings may also have implications for potential biomarkers. The current TGCT biomarkers of AFP, b-HCG, and LDH have a relatively low sensitivity and specificity (48). New tumor markers, particularly miRNAs have been actively developed $(49,50)$. The expression levels of HOXA10 are unlikely to be helpful since the absolute expression of HOXA10 largely remains unchanged in TGCT. However, as HOXA10 frequently loses its canonical functions in TGCT, it might be worthwhile to investigate whether downstream effectors of HOXA10 are amenable to non-invasive detections clinically.

\section{REFERENCES}

1. Hanna NH, Einhorn LH. Testicular cancer - discoveries and updates. N Engl J Med. (2014) 371:2005-16. doi: 10.1056/NEJMra1407550

2. Filippou P, Ferguson JE, Nielsen ME. Epidemiology of prostate and testicular cancer. Semin Intervent Radiol. (2016) 33:182-5. doi: 10.1055/s-0036-1586146

3. Znaor A, Lortet-Tieulent J, Jemal A, Bray F. International variations and trends in testicular cancer incidence and mortality. Eur Urol. (2014) 65:1095106. doi: 10.1016/j.eururo.2013.11.004

4. Rajpert-De Meyts E. Developmental model for the pathogenesis of testicular carcinoma in situ: genetic and environmental aspects. Hum Reprod Update (2006) 303-23. doi: 10.1093/humupd/dmk006

5. Williamson SR, Delahunt B, Magi-Galluzzi C, Algaba F, Egevad L, Ulbright TM, et al. The World Health Organization 2016 classification of testicular non-germ cell tumours: a review and update from the International Society of Urological Pathology Testis Consultation Panel. Histopathology (2017) 70:335-46. doi: 10.1111/his.13102

6. Abate-Shen C. Deregulated homeobox gene expression in cancer: cause or consequence? Nat Rev Cancer (2002) 2:777-85. doi: 10.1038/nrc907

7. Ferguson L, Agoulnik AI. Testicular cancer and cryptorchidism. Front Endocrinol. (2013) 4:32. doi: 10.3389/fendo.2013.00032

8. Sperger JM, Chen X, Draper JS, Antosiewicz JE, Chon CH, Jones SB, et al. Gene expression patterns in human embryonic stem cells and human pluripotent germ cell tumors. Proc Natl Acad Sci USA. (2003) 100:13350-5. doi: $10.1073 /$ pnas. 2235735100

9. Almstrup K, Hoei-Hansen CE, Wirkner U, Blake J, Schwager C, Ansorge W, et al. Embryonic stem cell-like features of testicular carcinoma in situ revealed by genome-wide gene expression profiling. Cancer Res. (2004) 64:4736-43. doi: 10.1158/0008-5472.CAN-04-0679

10. van Gurp RJ, Oosterhuis JW, Kalscheuer V, Mariman EC, Looijenga LH. Biallelic Expression of the H19 and IGF2 genes in human testicular germ cell tumors. J Natl Cancer Inst. (1994) 86:1070-5. doi: 10.1093/jnci/86.14.1070
In summary, we report that altered HOXA10 localization is associated with TGCT and that loss of nuclear HOXA10 canonical function results in attenuated TP53 signaling and increased cell cycling through regulating the G2/M checkpoint. Functional studies also further confirm that HOXA10 has antiproliferative activities in TGCT cells putatively via signaling pathways entailing TP53, cKit, STAT3, MAPK.

\section{AUTHOR CONTRIBUTIONS}

RC, HL, and YL contributed to the conception and design of the study as well as data acquisition and interpretation. RC drafted the manuscript. LF evaluated and scored IHC staining. MG, LN, and $\mathrm{XD}$ reviewed the manuscript critically. XD supervised and designed the study. All authors contributed to interpretation of findings, reviewed, edited, and approved the final manuscript.

\section{FUNDING}

This study was supported by the Canadian Institutes of Health Research (MOP137007 \& PTJ156150; XD).

\section{SUPPLEMENTARY MATERIAL}

The Supplementary Material for this article can be found online at: https://www.frontiersin.org/articles/10.3389/fonc. 2018.00594/full\#supplementary-material

11. Sievers S, Alemazkour K, Zahn S, Perlman EJ, Gillis AJM, Looijenga LHJ, et al. IGF2/H19 imprinting analysis of human germ cell tumors (GCTs) using the methylation-sensitive single-nucleotide primer extension method reflects the origin of GCTs in different stages of primordial germ cell development. Genes Chromosom Cancer (2005) 44:256-64. doi: 10.1002/gcc.20237

12. Skotheim RI, Lind GE, Monni O, Nesland JM, Abeler VM, Fosså SD, et al. Differentiation of human embryonal carcinomas in vitro and in vivo reveals expression profiles relevant to normal development. Cancer Res. (2005) 65:5588-98. doi: 10.1158/0008-5472.CAN-05-0153

13. Lind GE, Skotheim RI, Fraga MF, Abeler VM, Esteller M, Lothe RA. Novel epigenetically deregulated genes in testicular cancer include homeobox genes and SCGB3A1 (HIN-1). J Pathol. (2006) 210:441-9. doi: 10.1002/path.2064

14. Krumlauf R, Hill M. Hox genes in vertebrate development review. Cell (1994) 78:191-201. doi: 10.1016/0092-8674(94)90290-9

15. Benson GV, Nguyen TH, Maas RL. The expression pattern of the murine Hoxa-10 gene and the sequence recognition of its homeodomain reveal specific properties of Abdominal B-like genes. Mol Cell Biol. (1995) 15:1591601. doi: 10.1128/MCB.15.3.1591

16. Shen WF, Montgomery JC, Rozenfeld S, Moskow JJ, Lawrence HJ, Buchberg a $\mathrm{M}$, et al. AbdB-like Hox proteins stabilize DNA binding by the Meis1 homeodomain proteins. Mol Cell Biol. (1997) 17:6448-58. doi: 10.1128/MCB.17.11.6448

17. Shen WF, Rozenfeld S, Lawrence HJ, Largman C. The Abd-B-like Hox homeodomain proteins can be subdivided by the ability to form complexes with Pbxla on a novel DNA target. J Biol Chem. (1997) 272:8198-206. doi: 10.1074/jbc.272.13.8198

18. Lu Y, Goldenberg I, Bei L, Andrejic J, Eklund EA. HoxA10 represses gene transcription in undifferentiated myeloid cells by interaction with histone deacetylase 2. J Biol Chem. (2003) 278:47792-802. doi: 10.1074/jbc.M305885200

19. Yoshida H, Broaddus R, Cheng W, Xie S, Naora H. Deregulation of the HOXA10 homeobox gene in endometrial carcinoma: role 
in epithelial-mesenchymal transition. Cancer Res. (2006) 66:889-97. doi: 10.1158/0008-5472.CAN-05-2828

20. Guo L, Ding G, Xu W, Ge H, Jiang Y, Chen X, et al. MiR-135a-5p represses proliferation of HNSCC by targeting HOXA10. Cancer Biol Ther. (2018) 4047:1-28. doi: 10.1080/15384047.2018.1450112

21. Chu MC, Selam FB, Taylor HS. HOXA10 regulates p53 expression and matrigel invasion in human breast cancer cells. Cancer Biol Ther. (2004) 3:568-72. doi: 10.4161/cbt.3.6.848

22. Yu Y, Yang O, Fazli L, Rennie PS, Gleave ME, Dong X. Progesterone receptor expression during prostate cancer progression suggests a role of this receptor in stromal cell differentiation. Prostate (2015) 75:1043-50. doi: $10.1002 /$ pros. 22988

23. Li H, Xie N, Chen R, Verreault M, Fazli L, Gleave ME, et al. UGT2B17 expedites progression of castration-resistant prostate cancers by promoting ligand-independent AR signaling. Cancer Res. (2016) 76:6701-11. doi: 10.1158/0008-5472.CAN-16-1518

24. Xie N, Cheng $\mathrm{H}$, Lin D, Liu L, Yang O, Jia L, et al. The expression of glucocorticoid receptor is negatively regulated by active androgen receptor signaling in prostate tumors. Int J Cancer (2015) 136:E27-38. doi: 10.1002/ijc.29147

25. Yu Y, Liu L, Xie N, Xue H, Fazli L, Buttyan R, et al. Expression and function of the progesterone receptor in human prostate stroma provide novel insights to cell proliferation control. J Clin Endocrinol Metab. (2013) 98:2887-96. doi: 10.1210/jc.2012-4000

26. de Jong J, Stoop H, Gillis AJM, Hersmus R, van Gurp RJHL., van de Geijin G-JM, et al. Further characterization of the first seminoma cell line TCam-2. Genes Chromosomes Cancer (2008) 47:185-96. doi: 10.1002/gcc.20520

27. Liu L, Li Y, Xie N, Shynlova O, Challis JRG, Slater D, et al. Proliferative action of the androgen receptor in human uterine myometrial cells - A key regulator for myometrium phenotype programming. J Clin Endocrinol Metab. (2013) 98:218-27. doi: 10.1210/jc.2012-2451

28. Liu LL, Li H, Dargahi D, Shynlova O, Slater D, Jones SJM, et al. HoxA13 regulates phenotype regionalization of human pregnant myometrium. J Clin Endocrinol Metab. (2015) 100:E1512-22. doi: 10.1210/jc.2015-2815

29. Li Y, Donmez N, Sahinalp C, Xie N, Wang Y, Xue H, et al. SRRM4 drives neuroendocrine transdifferentiation of prostate adenocarcinoma under androgen receptor pathway inhibition. Eur Urol. (2017) 71:68-78. doi: 10.1016/j.eururo.2016.04.028

30. Chen R, Li Y, Buttyan R, Dong X. Implications of PI3K / AKT inhibition on REST protein stability and neuroendocrine phenotype acquisition in prostate cancer cells. (2017) 8:84863-76. doi: 10.18632/oncotarget.19386

31. Li W, Turner A, Aggarwal P, Matter A, Storvick E, Arnett DK, et al. Comprehensive evaluation of AmpliSeq transcriptome, a novel targeted whole transcriptome RNA sequencing methodology for global gene expression analysis. BMC Genomics (2015) 16:1069. doi: 10.1186/s12864-015-2270-1

32. Love MI, Huber W, Anders S. Moderated estimation of fold change and dispersion for RNA-seq data with DESeq2. Genome Biol. (2014) 15:550. doi: 10.1186/s13059-014-0550-8

33. Elbendary AA, Cirisano FD, Evans AC, Davis PL, Iglehart JD, Marks JR, et al. Relationship between p21 expression and mutation of the p53 tumor suppressor gene in normal and malignant ovarian epithelial cells. Clin Cancer Res. (1996) 2:1571-5.

34. Foster B, Zaidi D, Young T, Mobley M, Kerr B. CD117/c-kit in cancer stem cell-mediated progression and therapeutic resistance. Biomedicines (2018) 6:e31. doi: 10.3390/biomedicines6010031

35. Bael NS, Swanson MJ, Vassilev A, Howard BH. Human histone deacetylase SIRT2 interacts with the homeobox transcription factor HOXA10. J Biochem. (2004) 135:695-700. doi: 10.1093/jb/mvh084
36. Eklund EA, Jalava A, Kakar R. Tyrosine phosphorylation of HoxA10 decreases DNA binding and transcriptional repression during interferon $\gamma$-induced differentiation of myeloid leukemia cell lines. J Biol Chem. (2000) 275:2011726. doi: 10.1074/jbc.M907915199

37. Kakar R, Kautz B, Eklund EA. JAK2 is necessary and sufficient for interferon gamma-induced transcription of the gene encoding gp91 PHOX. J Leukoc Biol. (2005) 77:120-7. doi: 10.1189/jlb.0704429

38. Eklund EA, Goldenberg I, Lu Y, Andrejic J, Kakar R. SHP1 protein-tyrosine phosphatase regulates HoxA10 DNA binding and transcriptional repression activity in undifferentiated myeloid cells. J Biol Chem. (2002) 277:36878-88. doi: 10.1074/jbc.M203917200

39. Litchfield K, Levy M, Orlando G, Loveday C, Law PJ, Migliorini G, et al. Identification of 19 new risk loci and potential regulatory mechanisms influencing susceptibility to testicular germ cell tumor. Nat Genet. (2017) 49:1133-40. doi: 10.1038/ng.3896

40. Taylor WR, Stark GR. Regulation of the G2/M transition by p53. Oncogene (2001) 20:1803-15. doi: 10.1038/sj.onc. 1204252

41. Burger H, Nooter K, Boersma AW, Kortland CJ, Stoter G. Expression of p53, Bcl-2 and Bax in cisplatin-induced apoptosis in testicular germ cell tumour cell lines. Br J Cancer (1998) 77:1562-7. doi: 10.1038/bjc.1998. 257

42. Arozarena I, Wellbrock C. Overcoming resistance to BRAF inhibitors. Ann Transl Med. (2017) 5:387. doi: 10.21037/atm.2017.06.09

43. Damjanov I, Horvat B, Gibas Z. Retinoic acid-induced differentiation of the developmentally pluripotent human germ cell tumor-derived cell line, NCCIT. Lab Invest. (1993) 68:220-32.

44. Andrews PW. Human teratocarcinomas. BBA Rev Cancer (1988) 948:17-36.

45. Wood HM, Elder JS. Cryptorchidism and testicular cancer: separating fact from fiction. J Urol. (2009) 181:452-61. doi: 10.1016/j.juro.2008.10.074

46. Feng S, Bogatcheva NV, Truong A, Engel W, Adham IM, Agoulnik AI. Over expression of insulin-like 3 does not prevent cryptorchidism in GNRHR or HOXA10 deficient mice. J Urol. (2006) 176:399-404. doi: 10.1016/S0022-5347(06)00519-2

47. Pettersson A, Richiardi L, Nordenskjold A, Kaijser M, Akre O. Age at surgery for undescended testis and risk of testicular cancer. $N$ Engl J Med. (2008) 356:1835-41. doi: 10.1056/NEJMoa067588

48. Murray MJ, Huddart RA, Coleman N. The present and future of serum diagnostic tests for testicular germ cell tumours. Nat Rev Urol. (2016) 13:71525. doi: 10.1038/nrurol.2016.170

49. Syring I, Bartels J, Holdenrieder S, Kristiansen G, Müller SC, Ellinger J. Circulating serum miRNA (miR-367-3p, miR-371a-3p, miR-372-3p and miR373-3p) as biomarkers in patients with testicular germ cell cancer. J Urol. (2015) 193:331-7. doi: 10.1016/j.juro.2014.07.010

50. Murray MJ, Turnbull C. Testicular cancer in 2017: sequencing advances understanding. Nat Rev Urol. (2018) 15:79-80. doi: 10.1038/nrurol.2017.209

Conflict of Interest Statement: The authors declare that the research was conducted in the absence of any commercial or financial relationships that could be construed as a potential conflict of interest.

Copyright $\odot 2018$ Chen, Li, Li, Fazli, Gleave, Nappi and Dong. This is an open-access article distributed under the terms of the Creative Commons Attribution License (CC $B Y)$. The use, distribution or reproduction in other forums is permitted, provided the original author(s) and the copyright owner(s) are credited and that the original publication in this journal is cited, in accordance with accepted academic practice. No use, distribution or reproduction is permitted which does not comply with these terms. 